Pacific Journal of Mathematic 


\title{
LOCAL ANALYTIC EXTENSIONS OF THE RESOLVENT
}

\author{
JACK D. GRAY
}

\begin{abstract}
Consider an endomorphism $T$, (that is, a bounded, linear transformation) on a (complex) Banach space $X$ to itself. As usual, let $R(\lambda, T)=(\lambda 1-T)^{-1}$ be the resolvent of $T$ at $\lambda \in \rho(T)$. Then it is known that the maximal set of holomorphism of the function $\lambda \rightarrow R(\lambda, T)$ is the resolvent set $\rho(T)$. However, it can happen that for some $x \in X$, the $X$-valued function $\lambda \rightarrow R(\lambda, T) x$ has analytic extensions into the spectrum $\sigma(T)$ of $T$. Using this fact we shall, in $\S 1$, localize the concept of the spectrum of an operator. In sections 2,3 and 4 we investigate, quite thoroughly, the structural properties of this concept. Finally, in $\S 5$, the results of the previous sections will be utilized to construct a local operational calculus which will then be applied to the study of abstract functional equations.
\end{abstract}

1. The localization of the spectrum. We begin by making the following remarks. For an $X$-valued function $u$ to be analytic on some open subset $K$ of a Riemann surface, it is necessary and sufficient that for each continuous linear functional $x^{*}$ in some determining manifold for $X$, ([5],34), the complex-valued function $x^{*} u$ be analytic in $K . K$ will be called the maximal set of analyticity of $u$, if each accessible point of the boundary of $K$ is a singular point of $u$.

Now let $T: D_{T} \subseteq X \rightarrow X$ be an arbitrary linear transformation on its domain $D_{T}$, and suppose that $x \in X$.

Definition 1.1. The local resolvent set of $T$ at $x, \rho(x, T)$, is that set of points $\zeta \in C$-the complex plane - for which there is a neighbourhood $\mathrm{N}$ of $\zeta$, and an analytic function $u: N \rightarrow X$ which satisfies

$$
\lambda u(\lambda)-T u(\lambda)=x
$$

for all $\lambda \in N$.

Definition 1.2. The local spectrum $\sigma(x, T)$ of $T$ at $x$ is the complement in $C$ of $\rho(x, T)$.

Definition 1.3. The local spectral radius of $T$ at $x$ is given

$$
r(x, T)=\sup _{\lambda \in \sigma(x, T)}|\lambda| .
$$

DeFinition 1.4. Any function $u$ as given in Definition 1.1 will 
be called an (analytically) extended resolvent of $T$ at $x$, on $N$.

In a slightly different form these concepts have been introduced by Dunford [1], and have been used by him in his characterization of spectral operators. However, in the following theorem we shall show that the two concepts are equivalent, whenever both are defined.

Theorem 1.2. Suppose $N$ is an open set with $N \cap \rho(T) \neq 0$. Then $u$ is an extended resolvent of $T$ at $x$ on $N$, if and only if $u$ $i s$ an analytic continuation of $R(\cdot, T) x$ into $N$.

Proof. Suppose first that $u$ is an analytic continuation of $R(\cdot, T) x$ to $N$, so that $u$ is analytic on $N \cap \rho(T)$, and $u(\lambda)=R(\lambda, T) x$ if $\lambda \in \rho(T)$. Because of the low of permanance of functional equations, the first resolvent equation shows us that $u$ must satisfy

$$
[1-(\mu-\lambda) R(\mu, T)] u(\lambda)=R(\mu, T) x,
$$

at least for $\mu \in \rho(T)$ and $\lambda \in N \cap \rho(T)$. Thus, operating on this equation by $\mu 1-T$, we arrive at $(\lambda 1-T) u(\lambda)=x$, and so, by Definition 1.1, $u$ is an extended resolvent of $T$ at $x$. Conversely, if $u$ is an extended resolvent of $T$ at $x$, analytic on $N \cap \rho(T)$, then, with $T_{\lambda}=\lambda 1-T$, we have $T_{\lambda} u(\lambda)=x$ and $T_{\lambda} R(\lambda, T) x=x$, at least for $\lambda \in \rho(T)$. So, $T_{\lambda}(u(\lambda)-R(\lambda, T) x)=0$, and this obviously implies that $u(\lambda)=R(\lambda, T) x$ if $\lambda \in \rho(T)$. Therefore, $u$ is an analytic continuation of the resolvent of $T$ into $N$.

Now, the resolvent $R(\cdot, T)$ has as domain, the (not necessarily connected) open set $\rho(T)$, and so $R(\cdot, T)$ is only locally holomorphic on $\rho(T)$. That is $R(\cdot, T)$ is holomorphic on each component of $\rho(T)$. Furthermore, it is known that $\rho(T)$ is the maximal set of (local) holomorphism of $R(\cdot, T)$. With this notation we now observe the following deduction from Theorem 1.2.

COROLlary 1.3. The union of those components of $\rho(x, T)$ which contain points of $\rho(T)$, is the maximal set of local holomorphism of $R(\cdot, T) x$.

In general, an extended resolvent of $T$ at $x$ on an open set $N$ will not be an analytic continuation of $R(\cdot, T) x$, as $N$ may be entirely contained in $\sigma(T)$, so that $R(\cdot, T)$ will not be defined on $N$.

Remarks. (i) Suppose that $B$ is an arbitrary (complex) Banach algebra with identity. Then, by considering the regular representations of $B$ as the algebra $B(X)$ of endomorphisms on some Banach space 
$X$, the above definitions remain intact, and so they provide a generalization of the concept of the spectrum for these algebras.

(ii) In [4], we have considered not only analytic extensions of the resolvent, but also continuous and weakly continuous solutions of equation (1.1). In particular, it was found when these three extensions coincide.

2. Topological properties of the local spectrum. We shall now see that the (topological) properties of the local spectrum of $T$ at $x$ closely resemble those of $\sigma(T)$. We first of all prove a result which will be useful in a later section.

Lemma 2.1. Suppose that $A \in B(X)$ commutes with $T$. Then for all $x \in X, \sigma(A x, T) \subseteq \sigma(x, T)$. If further, $A$ is a regular element $B(X)$, then equality holds.

Proof. Suppose that $\lambda \in \rho(x, T)$. Then there is a neighbourhood $N$ of $\lambda$, and a function $u: N \rightarrow X$, analytic on $N$, such that $\lambda u(\lambda)-T u(\lambda)=x$ for all $\lambda \in N$. But then

$$
A x=\lambda A u(\lambda)-A T u(\lambda)=\lambda A u(\lambda)-T A u(\lambda),
$$

and therefore, as $A$ is continuous, $A u$ is analytic on $N$, and from the above equation, we see therefore that $A u$ is an extended resolvent of $T$ at $A x$, on $N$. Hence $\sigma(A x, T) \leqq \sigma(x, T)$. If $A^{-1}$ is also bounded, then, as $A^{-1}$ also commutes with $T$, a similar argument yields $\sigma(A x, T) \supseteqq \sigma(x, T)$. The result follows from these inclusions. In particular, for each nonzero $\alpha \in C$ we have $\sigma(\alpha x, T)=\sigma(x, T)$.

Lemma 2.2. If $T \in B(X)$, then $r(x, T)=\lim \sup _{n \rightarrow \infty}\left\|T^{n} x\right\|^{1 / n}$. In general the limit does not exist.

Proof. From Theorem 1.1 and Definition 1.4, $r(x, T) \leqq r(T)$ for each $x \in X$. Now, for $|\lambda|>r(x, T)$ there is an analytic continuation of the resolvent $u$, which is represented by an absolutely convergent Laurent series about $\infty$, thus $u(\lambda)=\sum_{0}^{\infty} a_{n} \lambda^{-n}$, with $a_{n}=a_{n}(x) \in X$. But for $|\lambda|>r(T) \geqq r(x, T)$, because of the Neumann expansion of the resolvent the series

$$
\lambda^{-1} \sum_{0}^{\infty} \lambda^{-n} T^{n} x
$$

converges absolutely, and represents an extended resolvent for each $x$. Therefore, by the uniqueness theorem for vectorvalued analytic functions, the two series are identical. It is now easily seen that 
the radius of convergence of (2.1) is $r(x, T)$, and the first part of the lemma now follows from the Hadamard formula.

As for the second part, let $X$ be the Banach space of all bounded (complex) sequences $x=\left(x_{1}, x_{2}, \cdots\right)$ with norm $\|x\|=\sup _{n \geqq 1}\left|x_{n}\right|$. For a given sequence of real numbers $\left\{a_{n}\right\}$, with $0<a_{n} \leqq 1$, define the shift $T$ on $X$ by $T x=\left(0, a_{1} x_{1}, a_{2} x_{2}, \cdots\right)$. Then $\|T x\|=$ $\sup _{n \geqq 1}\left|a_{n} x_{n}\right| \leqq\|x\|$, for all $x \in X$, so that $T \in B(X)$. With $x=$ $(1,0,0, \cdots) \in X$ we find that $T^{n} x=\left(0,0, \cdots, 0, a_{1} a_{2} \cdots a_{n}, 0,0, \cdots\right)$ for each integer $n$, and so $\left\|T^{n} x\right\|^{1 / n}=\left(a_{1} a_{2} \cdots a_{n}\right)^{1 / n}$. Now make the following choice, $a_{n}=2^{-n}$ if $n=10^{N}$ for some integer $N>0$, and $\mathrm{a}_{n}=1$ otherwise. We then find that if $n \rightarrow \infty$ through powers of 10 only, $\left\|T^{n} x\right\|^{1 / n} \rightarrow 2^{-10 / 9}<1$, and otherwise, $\left.\left\|T^{n} x\right\|\right|^{1 / n} \rightarrow 1$. So the limit infimum and the limit supremum are not equal, and this completes the proof.

We come now to the main result of this section,

THEOREM 2.3. For each $x \in X, \sigma(x, T)$ is closed. If further, $T \in B(X)$, then $\sigma(x, T)$ is compact, and it is empty if and only if $x=0$.

Proof. By Definition 1.1, the set $\rho(x, T)$ is open, and so $\sigma(x, T)$ is closed. We have already observed in Theorem 1.1 that $\sigma(x, T) \subseteq$ $\sigma(T)$ for all $x \in X$. So if $T$ is bounded, $\sigma(T)$ is also bounded, and therefore $\sigma(x, T)$ is compact. Suppose now that the local spectrum of $T \in B(X)$ at $x$ is empty. Then, because of Lemma 2.2, we see that the series (2.1) converges to an extended resolvent for all nonzero values of $\lambda$. Thus $u(\lambda) \rightarrow 0$ as $|\lambda| \rightarrow \infty$. Further, in this case, $u$ is an entire function. Therefore, by the Liouville theorem for vector-valued analytic functions, $u$ is identically zero. But this would imply that $0=\lambda u-T u=x$. The converse of this proposition being obvious, the theorem is proven.

We mention the following facts. As in classical spectral theory, the local spectrum of an unbounded operator may be either bounded, or unbounded. This is illustrated by the following example. Let $X$ be the Banach space of bounded sequences, as in Lemma 2.2. Let $D_{T}=\left\{x \in X:\left|x_{n}\right|=0\left(n^{-1}\right)\right.$ as $\left.n \rightarrow \infty\right\}$ be the domain of the operator $T$ defined by $T\left(x_{1}, x_{2}, \cdots\right)=\left(x_{1}, 2 x_{2}, \cdots, n x_{n}, \cdots\right)$. Then $T$ is certainly unbounded, and $\sigma(T)$ is the set of positive integers. If, however, we choose $x$ so that for $\mathrm{n}>N, x_{n}=0$, then $\sigma(x, T) \subseteq\{1,2, \cdots, N\}$, which is bounded. Similarly, if we choose $x \in X$ so that for $n>N, x_{n} \neq 0$, then $\sigma(x, T) \supseteqq\{N, N+1, \cdots\}$. Along this line one can construct operators $T$ (unbounded of course), for which $\sigma(x, T)=\dot{\phi}$ for all $x$; and operators for which $\rho(x, T)=\phi$. In the first case we choose an unbounded operator for which $\sigma(T)$ is empty, (cf: [2], 605), then, by 
Theorem 1.1, $\sigma(x, T)=\phi$ for each $x \in X$. Secondly, let $X$ be the space $C[0, \infty]$, and let $T$ be the operation of differentiation on the domain $\left\{x: x^{\prime} \in C[0, \infty]\right.$ and $\left.x(0)=0\right\}$. Choose $x(t)=e^{-t}$. Then we find that the local spectrum of $T$ at $x$ is the entire complex plane, so that $\rho(x, T)$ is empty.

In later sections it will become important to know something about the connectedness of the local spectrum, but, as the following theorem shows, we can say nothing general about this.

THEOREM 2.4. Compactness is the only topological property shared by all local spectra.

Proof. Let $X$ be the Banach space of all bounded, complex-valued functions on $C$, with the usual pointwise algebraic operations, and with the topology induced by the supremum norm. For a fixed $a \in X$, define the mapping $T: X \rightarrow X$ by $(T u)(z)=a(z) u(z)$ for $u \in X$ and all $z \in C$; so that $T \in B(X)$. The local spectrum of $T$ at $x$ is the complement of the set of points $\lambda$ at which there is an analytic function $\lambda \rightarrow u(\lambda)$ which satisfies $\lambda u-a u=x$. But, as the solution of this equation is $(u(\lambda))(z)=x(z) /(\lambda-a(z))$, this set is the closure of the range $\Gamma=\{a(z): z \in C \backslash Z\}$, where $Z$ is the set of zeroes of $x$. Thus $a$ and $x$ may be chosen so that $\mathrm{Cl}(\Gamma)$ is any given closed, bounded subset of $C$. Hence, any compact set is the local spectrum of a suitable endomorphism $T$ on $X$, at a suitable point $x \in X$.

We now examine the relationship between $\sigma(T)$ and the local spectra of $T$. In particular, the next result tells us how many of the local spectra are required to cover $\sigma(T)$.

TheoREM 2.5. If $T \in B(X)$ and $X_{0} \subseteq X$ is a subspace of the second category, then $\mathrm{U}_{x \in X_{0}} \sigma(x, T)=\sigma(T)$.

Proof. We have from Theorem 1.1 that $\cup \sigma(x, T) \subseteq \sigma(T)$, the union being taken over all $x \in X_{0}$ - some subspace of the second category in $X$. To prove the theorem we will show that this inclusion may be reversed. Toward this end assume that $\alpha \in \bigcap_{x \in X_{0}} \rho(x, T)$. Then, for all $x \in X_{0}$, there is a neighbourhood of $\alpha$ and an analytic $X$-valued function $\lambda \rightarrow u(\lambda, x)$ which satisfies $\lambda u(\lambda, x)-T u(\lambda, x)=x$. Next, define the mapping $P: X_{0} \rightarrow X$ via $P x=u(\alpha, x)$, for $x \in X_{0}$. Certainly if $x, y \in X_{0}$, then $P(x+y)=P(x)+P(y)$ because $\alpha \in \rho(x, T) \cap \rho(y, T)$. Thus $P$ is a linear transformation on $X_{0}$ to $X$. Now, for each $x \in X_{0}$ we have $(\alpha 1-T) P x=x$, and so

$$
\|x\|=\|(\alpha 1-T) P x\| \leqq(|\alpha|+\|T\|)\|P x\| ;
$$


that is, there exists a constant $M>0$ for which $\|P x\| \geqq M\|x\|$ for each $x \in X_{0}$. Thus $P$ has a bounded inverse $P^{-1}$ with domain $X_{1}=$ sp $\{u(\alpha, x): x \in X\}$-the subspace generated by $u(\alpha, x)$. It is plainly seen that this inverse $P^{-1}$ is in fact the restriction of $\alpha 1-T$ to $X_{1}$. As $P^{-1}$ is bounded on its domain, it can be extended, by continuity, to a bounded operator on $\mathrm{Cl}\left(X_{1}\right)$-the closure of $X_{1}$. Therefore, this extension $P^{-1}$ is necessarily closed on its domain, so its inverse $\left(P^{-1}\right)^{-1}=P: X_{0} \rightarrow \mathrm{Cl}\left(X_{1}\right)$ is also closed. Finally, as $X_{0}$ is of the second category in $X$, we invoke the closed graph theorem to conclude that the domain of $P$ is actually all of $X$, and that $P$ is bounded. Thus we have shown that $\alpha 1-T$ has a bounded inverse with domain $X$, and this condition necessarily implies that $\alpha$ belongs to the resolvent set of $T$. From this statement the theorem follows.

Corollary 2.6. (Dunford [1]). If $T \in B(X)$, then $\bigcup_{x \in X} \sigma(x, T)=$ $\sigma(T)$.

This corollary is a direct consequence of Theorem 2.5 taken in conjunction with the Baire category theorem. Even without completeness, if $X$ is only a normed linear space, and if $T$ is any linear transformation, the conclusion of Corollary 2.6 is still valid, as $\sigma(T)$ is the union, over all $x \in X$ and all $x^{*}$ in some determining manifold for $X$, of the sets of singularities of $x^{*}(R(\cdot, T) x)$; whereas $\sigma(x, T)$ is the union, over all $x^{*}$ in this determining manifold, of the sets of singularities of $x^{*}(R(\cdot, T) x)$.

In particular cases the result of Theorem 2.5 may be improved upon, in that it may require less of the local spectra to cover $\sigma(T)$. Suppose, for example, that $T$ is quasi-nilpotent, (or, more generally, that $T \in B(X)$ has only a single point in its spectrum), then, by the third part of Theorem 2.3, $\sigma(x, T)=\sigma(T)$ for each nonzero $x \in X$. In fact, we are led to make the following conjecture regarding the existence of such extremal vectors.

Conjecture. If $T \in B(X)$, there is an $x \in X$ for which $\sigma(x, T)=\sigma(T)$.

To support this conjecture we state the:

THEOREM 2.7. For each point $\zeta \in \mathrm{Bd} \sigma(T)$, there is a set $X_{\zeta}$, of the second category in $X$, for which $\zeta \in \mathrm{Bd} \sigma(x, T)$ for all $x \in X_{\zeta}$.

Proof. As $\zeta$ belongs to the boundary of $\sigma(T)$ there is a sequence $\left\{\zeta_{n}\right\}$ in the resolvent set of $T$, such that $\zeta_{n} \rightarrow \zeta$ as $n \rightarrow \infty$. Now let $R_{n}=R\left(\zeta_{n}, T\right)$ for $n=1,2, \cdots$. Then each $R_{n}$ is an endomorphism on $X$. But, because of Theorem VII 3.3 of [2], we have that 


$$
\left\|R_{n}\right\| \geqq 1 / d\left(\zeta_{n}, \sigma(T)\right) \rightarrow \infty
$$

as $n \rightarrow \infty$. Thus, by the principle of the condensation of singularities, ([2], 81) there is a set $X_{\zeta}$, of the second category in $X$, for which $\left\|R_{n} x\right\| \rightarrow \infty$ as $n \rightarrow \infty$, for each $x \in X_{\zeta}$. But by Definition 1.1. and the definition of analyticity, this means that $\zeta$ is a singular point of the function $R(\cdot, T) x$, and so $\zeta \in \mathrm{Bd} \sigma(x, T)$ for each vector $x \in X_{\zeta}$.

3. The analysis of extended resolvents. Our Definition 1.4 of an extended resolvent $u$ of $T$ at $x$, was a purely local definition, in that these extensions were defined only on open subsets of $\rho(x, T)$. Suppose in fact, that there is a component of the local resolvent set of $T$ at $x$ which is entirely contained in $\sigma(T)$. Let $u_{1}$ and $u_{2}$ be two extended resolvents of $T$ at $x$, analytic on open sets $N_{1}, N_{2} \sqsubseteq \kappa$. Then it may happen that $u_{1}$ and $u_{2}$ differ on the intersection $N_{1} \cap N_{2}$. This is illustrated by an example due to Stampfli ([8], 288). If, however, $\kappa$ and $\rho(T)$ are connected, then, because of Theorem 1.2, there is a unique analytic extended resolvent defined on $\kappa$.

This statement leads us to the following.

DEFinition 3.1. If there is only one extended resolvent of $T$ at $x$, analytic on $\rho(x, T)$, we shall say, (following Dunford [1]), that $T$ has the single-valued extension property at $x$.

This property has been of fundamental importance in Dunford's work on the characterization of spectral operators; and certain sufficient conditions for $T$ to have this property (for all $x \in X$ ), were given by him. (Cf: Lemma 1 on page 251, and Lemma 4 on page 254 of [1].) These conditions will be contained in the next theorem. In this theorem $T$ will denote a (not necessarily bounded) linear transformation on $X$, and $P \sigma(T)$ will denote its point spectrum.

THEOREM 3.1. A sufficient condition for $T$ to have the singlevalued extension property at $x$, is that for each component $\kappa$ of $\rho(x, T)$, the set $\kappa \backslash P \sigma(T)$ has a limit point in $\kappa$.

Proof. We note first that as $\rho(x, T)$ is open, and as $C$ is locally connected, each component of $\rho(x, T)$ is open. Now let $u$ and $\hat{u}$ be any two extensions of the resolvent of $T$ at $x$, analytic in $\rho(x, T)$. Let $\kappa$ be an arbitray component of the local resolvent set. Then we find that for all $\lambda \in \kappa, T_{\lambda} \hat{u}(\lambda)=x=T_{\lambda} u(\lambda)$, so $T_{\lambda}(\hat{u}(\lambda)-u(\lambda))=0$. From this equation we see that $\hat{u}(\lambda)=u(\lambda)$ if $\lambda \in \kappa \backslash P \sigma(T)$, and $\hat{u}(\lambda)=$ $u(\lambda)+\xi(\lambda)$ if $\lambda \in \kappa_{n} P \sigma(T)$. Here $\xi(\lambda)$ is an element of the null-space of $T_{\lambda}$, and the function $\lambda \rightarrow \xi(\lambda)$ is necessarily analytic on $\kappa$. Now, if the above hypothesis is satisfied, $\kappa \backslash P \sigma(T)$ must contain a sequence 
$\left\{\zeta_{n}\right\}$ with $\zeta_{n} \rightarrow \zeta \in \kappa$, and we thus see that $\xi\left(\zeta_{n}\right)=0$ for $n=1,2, \cdots$, and therefore, by the uniqueness theorem for vector-valued analytic functions, $\xi=0$ throughout $\kappa$. Hence $\hat{u}(\lambda)=u(\lambda)$ for all $\lambda \in \kappa$.

We observe that for $T$ to have the single-valued extension property at $x$ it is not necessary for the above condition to be satisfied. For let $X$ be the Banach space of all bounded, complex-valued functions on $C$, as in Theorem 2.4. Let $T \in B(X)$ be the operation of multiplication by $a \in X$, where

$$
a(z)=\left\{\begin{array}{lll}
z & \text { if } & |z| \leqq 1 \\
1 & \text { if } & |z|>1
\end{array} .\right.
$$

Define $x \in X$ by

$$
x(z)=\left\{\begin{array}{l}
z \text { if }|z|=1 \\
0 \text { otherwise }
\end{array} .\right.
$$

Then, from Theorem 2.4, we find easily that $\sigma(T)=\bar{\Delta}(0,1)$-the closed unit disc; $P \sigma(T)=\Delta(0,1)$ - the open unit disc; and the local resolvent set of $T$ at $x$ consists of the two components $\Delta(0,1)$, and the complement of $\bar{\Delta}(0,1)$.

So, in the unbounded component of $\rho(x, T)$, the conditions of Theorem 3.1 are not satisfied, but still there is only one extended resolvent of $T$ at $x$, analytic in $\Delta(0,1)$. As if there were two such extensions, their difference would be a nonzero function $\xi$ for which $\xi(\lambda)$ belongs to the null-space of $T_{\lambda}$ for each $\lambda \in \Delta(0,1)$. However, a simple computation shows that if $|\lambda|<1$, the null-space of $T_{2}$ is the one-dimensional subspace generated by the element

$$
(\xi(\lambda))(z)=\left\{\begin{array}{lll}
0 & \text { if } & z \neq \lambda \\
1 & \text { if } & z=\lambda
\end{array} .\right.
$$

and so the function $\lambda \rightarrow \xi(\lambda)$ is not an analytic $X$-valued function on $\Delta(0,1)$.

Corollary 3.2. If no component of $\rho(x, T)$ is contained in $\sigma(T)$, then $T$ has the single-valued extension property at $x$.

The spectral operators in [1] satisfied the conditions of:

Corollary 3.3. (Dunford). If $\sigma(T)$ is nowhere dense, then $T$ has the single-valued extension property for all $x \in X$.

Suppose now that $T$ does not have the single-valued extension property at $x$, so that in some component $\kappa$ of $\rho(x, T)$ there are two 
distinct analytic functions $u_{1}$ and $u_{2}$ which satisfy equation (1.1). It may happen that the values of $u_{1}$ and $u_{2}$ in $\kappa$ represent the different branches of a single analytic $u$. In this case we may, (by suitable cuts), define an open Riemann surface $\kappa^{*}$ in $\kappa$ on which $u$ is holomorphic. Then $\kappa^{*}$ will be a maximal domain of holomorphism of the unique analytic solution of equation (1.1), and so $T$ will have the singlevalued extension property at $x$, in $\kappa^{*}$. That this type of behaviour can occur is illustrated by Kakutani on page 185 of [5].

We conclude this section by exhibiting two examples which will both illustrate the theory, and provide counter-examples for it.

EXAMPLE 3.1. Let $J$ be the projection of the Hilbert space $X$ onto the closed subspace $M$, so that $J \in B(X), J^{2}=J$, and $X=M \oplus N$ is the direct sum decomposition of $X$. If we assume that $J$ is neither the identity, nor the zero projection, then $\sigma(J)=\{0,1\}$, and it is readily seen that

$$
R(\lambda, J)=\lambda^{-1} 1+\lambda^{-1}(\lambda-1)^{-1} J
$$

for all $\lambda \in \rho(J)$. From this we find that if $x \in M$ then $\sigma(x, J)=\{1\}$; if $x \in N, \sigma(x, J)=\{0\}$, and if $x$ is in neither of these two subspaces, that is, if $x=y+z$ with $0 \neq y \in M$ and $0 \neq z \in N$, then $\sigma(x, J)=\{0,1\}=$ $\sigma(J)$.

EXAMPLE 3.2. As our second example we consider the unilateral shift $U$ on the Hilbert space $l_{2}$ of square summable (complex) sequences, defined by $U\left(x_{1}, x_{2}, \cdots\right)=\left(0, x_{1}, x_{2}, \cdots\right) \in l_{2}$. Then, as noted by Sine, $([7], 335), \sigma(x, U)=\sigma(U)=$ the closed unit disc $\bar{J}(0,1)$, for each nonzero $x \in l_{2}$. This is most easily seen by considering the $l_{2}$ solutions of the difference equation

$$
\lambda u_{n+1}-u_{n}=x_{n},
$$

for an arbitrary vector $\left\{x_{n}\right\} \in l_{2}$.

4. The structure of the local spectrum (contd.). There is an intimate relation between the spectral properties of a bounded linear operator $T: X \rightarrow X$, and those of its adjoint $T^{*}: X^{*} \rightarrow X^{*}$, namely, $\sigma(T)=\sigma\left(T^{*}\right)$ and $R(\lambda, T)^{*}=R\left(\lambda, T^{*}\right)$ for all $\lambda \in \rho(T)=\rho\left(T^{*}\right)$. However, because our spectral concepts are localized at a given point of $X$, and as there is, in general, no canonical map from $X$ to the dual space $X^{*}$ of continuous linear functionals on $X$, we would not expect such satisfying relations to be true. Even when there is such a natural map, as, for example, when $X$ is a Hilbert space, we still do not find desirable properties, as the following example shows. Choose $T=U$ as in Example 3.2 of the previous section, then $\sigma(x, U)=\sigma(U)$ for all 
$x \neq 0$. In spite of this, the adjoint $U^{*}$ is such that its resolvent does have analytic extensions beyond $\rho\left(U^{*}\right)$. Here $U^{*}$ is the left unilateral shift on $l_{2}$ defined by $U^{*}\left(x_{1}, x_{2}, \cdots\right)=\left(x_{2}, x_{3}, \cdots\right)$. Now choose $x=\left\{x_{n}\right\} \in l_{2}$ to have only a finite (positive) number of nonzero components, so there is an integer $N$ with $n>N$ implying that $x_{n}=0$. Then it is readily seen that 0 has index $N$, and so $R\left(\lambda, U^{*}\right) x$ is a polynomial of degree $N$ in $1 / \lambda$. Furthermore, $\sigma\left(x, U^{*}\right)=\{0\}$ which is properly contained in $\sigma\left(U^{*}\right)$. But to disprove the possible conjecture that for suitable $x^{*} \in X^{*}, \sigma\left(x^{*}, T^{*}\right) \subseteq \sigma(x, T)$, we merely note that $U^{* *}=U$, and so $\sigma\left(x, U^{* *}\right) \supseteqq \sigma\left(x, U^{*}\right)$.

For certain special operators on Hilbert space, we can say something positive. However, first we need the

Lemma 4.1. Under extensions of $T$, the local spectra of $T$ suffer contractions.

Proof. Let $T^{\prime}$ be an extension of the linear transformation $T$ with domain $D_{T^{\prime}} \supseteqq D_{T}$. Suppose that $x \in X$, and let $\lambda_{0} \in \rho(x, T)$. Then we have an analytic function $u$ on a neighbourhood of $\lambda_{0}$, such that $\lambda u(\lambda)-T u(\lambda)=x$, and so $u(\lambda) \in D_{T}$, for all $\lambda$ in this neighbourhood. Further, $\lambda u(\lambda)-T^{\prime} u(\lambda)=x$, so that $\lambda \in \rho\left(x, T^{\prime}\right)$, and therefore $\sigma(x, T) \supseteqq$ $\sigma\left(x, T^{\prime}\right)$ for all $x \in X$. Under restrictions of $T$ the reverse effect is found.

THEOREM 4.2. If $T$ is symmetric, then $\sigma(x, T)=\sigma\left(x, T^{*}\right)$ for all $x \in X$.

Proof. By definition we know that the (Hilbert space) adjoint operator $T^{*}$ exists and is an extension of $T$. Hence, by Lemma 4.1, $\sigma(x, T) \supseteqq \sigma\left(x, T^{*}\right)$ for all $x \in X$. Thus, because of Corollary 2.6 we see that $\sigma(T)=\bigcup_{x \in T} \sigma(x, T) \supseteqq \bigcup_{x \in T} \sigma\left(x, T^{*}\right)=\sigma\left(T^{*}\right)=\bar{\sigma}(T)$ - where the bar denotes complex conjugation, that is, $\bar{\sigma}(T)=\{\bar{\lambda}: \lambda \in \sigma(T)\}$. However, this condition obviously implies that $\sigma(T)=\bar{\sigma}(T)$, which then implies that $\mathrm{U}_{x \in X} \sigma(x, T)=\mathrm{U}_{x \in X} \sigma\left(x, T^{*}\right)$, and so, because of what we have proven above, $\sigma(x, T)=\sigma\left(x, T^{*}\right)$ for each $x \in X$.

TheOREM 4.3. If $T$ is unitary, then $\sigma(x, T)=1 / \sigma\left(x, T^{*}\right)$ for all $x \in X$.

Proof. From the fundamental equation which defines an analytic extended resolvent $u$ of $T$ at $x$, we find that

$$
T^{*} x=\lambda T^{*} u(\lambda)-T^{*} T u(\lambda)=\lambda T^{*} u(\lambda)-u,
$$

as $T$ is unitary, and for all $\lambda \in \rho(x, T)$. So $\lambda^{-1} u(\lambda)-T^{*} u(\lambda)=-\lambda^{-1} T^{*} x$, 
which shows that $\lambda^{-1} \in \rho\left(-\lambda^{-1} T^{*} x, T^{*}\right)$. But $T^{*}$ commutes with $T$, and is a regular element of $B(X)$, and therefore, by Lemma 2.1, $\sigma\left(-\lambda^{-1} T^{*} x, T^{*}\right)=\sigma\left(x, T^{*}\right)$, so that $\lambda^{-1} \in \rho\left(x, T^{*}\right)$. Now, as $T \in B(X)$, $T^{* *}=T$, and hence $T^{*}$ is also unitary, so $\rho\left(x, T^{*}\right)=1 / \rho\left(x, T^{* *}\right)=$ $1 / \rho(x, T)$, and from this, and the above, we find that $\sigma(x, T)=$ $1 / \sigma\left(x, T^{*}\right)=\left\{1 / \lambda: \lambda \in \sigma\left(x, T^{*}\right)\right\}$, for all $x \in X$.

In the same vein, Stampfli, ([8], 291), has proven the

THEOREM 4.4. If $T$ is completely hyponormal, and if $\sigma(T)$ lies on an arc, then $\sigma(x, T) \supseteqq \bar{\sigma}\left(x, T^{*}\right)$ for all $x \in X$.

The strength of the hypotheses involved in Theorem 4.4 seem unavoidable in view of the next result. Before proving this however, we present some notation. Suppose that $T: D_{T} \subseteq X \rightarrow X$ is a linear operator, that $X$ is a Hilbert space, and that $u$ is an extended resolvent of $T$ at $x$ in $\rho(x, T)$. We denote by $M=M(x, T)$ the closed subspace generated by $\{u(\lambda): \lambda \in \rho(x, T)\}$.

TheOREM 4.5. Suppose that $T$ is densely defined in $X$, and that $M(x, T)$ reduces $T$, then $\sigma(x, T) \supseteqq \bar{\sigma}\left(x, T^{*}\right)$. If, further, $T$ has a closure $\hat{T}$, and if $M\left(x, T^{*}\right)$ reduces $\hat{T}$, then equality holds.

Proof. We have the existence of a function $u$, analytic on $\rho(x, T)$, and satisfying $x=\lambda u(\lambda)-T u(\lambda)$ in $\rho(x, T)$. So, for all $w \in X$,

$$
(x, w)=(\lambda u-T u, w)=\lambda(u, w)-(T u, w)=\lambda(u, w)-\left(u, T^{*} w\right),
$$

at least for those $w \in D_{T^{*}}$ - the domain of $T^{*}$. Therefore, for $\lambda \in \rho(x, T)$,

$$
\left(u(\lambda), \bar{\lambda} w-T^{*} w\right)=(x, w) .
$$

We now wish to show that in the Hilbert space $X$, for any sequence $\left\{a_{n}: n=0,1,2, \cdots\right\} \subseteq X$, and for a fixed element $x \in X$, we can define a sequence of vector $\left\{b_{n}: n=0,1,2, \cdots\right\} \subseteq X$ such that $\left(x, b_{n}\right)=\left(a_{n}, x\right)$, and $\left\|a_{n}\right\|=\left\|b_{n}\right\|$ for each integer $n$. To this end we use the fact that every Hilbert space $X$ is isometrically isomorphic to a Lebesgue space $L^{2}(S, \Sigma, \mu)$, for some measure space $S$, a $\sigma$-ring of subsets $\Sigma$ of $S$, and a measure $\mu$ on $\Sigma$ (cf: [2], 349). Under this isomorphism we identify $x$ with the square $\mu$-integrable function $x(\cdot)$ on $S$, and similarly with the sequence $\left\{a_{n}\right\}$, so that for each positive integer $n$, we may certainly define an $L^{2}$ function $b_{n}$ for which

$$
\int_{S} x(t) \bar{b}_{n}(t) d \mu=\int_{S} a_{n}(t) \bar{x}(t) d \mu .
$$


Because, if $\Omega \in \Sigma$ is the subset of $S$ on which $x$ vanishes, we define $b_{n}(t)=\bar{a}_{n}(t) x(t) / \bar{x}(t)$ if $t \notin \Omega$; and for $t \in \Omega$, we define $b_{n}(t)=\bar{a}_{n}(t)$. Then certainly each $b_{n}$ is square $\mu$-integrable on $S$, and by construction, $\left|a_{n}(t)\right|=\left|b_{n}(t)\right|$ for all $t \in S$. Thus, as the above isomorphism is an isometry, we conclude that $\left\|a_{n}\right\|=\left\|b_{n}\right\|$ for all integers $n$, and (4.2) implies that $\left(x, b_{n}\right)=\left(a_{n}, x\right)$.

Now, as $u$ is analytic in $\rho(x, T)$, each element of $u$ has a Laurent expansion of the form

$$
\sum_{0}^{\infty} a_{n}(z-\alpha)^{n}
$$

for some $\alpha \in C$, where the series (4.3) converges absolutely for

$$
|z-\alpha|<1 / \lim \sup _{n \rightarrow \infty}\left\|a_{n}\right\|^{1 / n}=\rho>0 .
$$

Next, choose a sequence $\left\{b_{n}\right\} \subseteq X$ as above, and define the function $w$ by

$$
w(z)=\sum_{0}^{\infty} b_{n}(z-\bar{\alpha})^{n} .
$$

This series then converges absolutely for $|z-\bar{\alpha}|<\rho$, and so in this disc it defines an analytic function. However, by the choice of the sequence of co-efficients in (4.4), $w$ also satisfies the equation

$$
(x, w)=(u, x) \text {. }
$$

Thus, from the above equations, we have constructed a function $w$, analytic at $\bar{\alpha}$, and satisfying (4.1). Thus, there is an element $\xi \in M^{\perp}-$ the orthocomplement of $M(x, T)$, for which

$$
x+\xi=\bar{\lambda} w(\lambda)-T^{*} w(\lambda)
$$

for $\lambda \in \rho(x, T)$. However, as $M$ reduces $T$, the projection $J: X \rightarrow M$ commutes with $T$ on $D_{T} \supseteqq M$, and further, the subspace $M^{\perp}$ is the null-space of $J$, so that $J \xi=0$. Furthermore, we know that $M$ is invariant under $T$, and from this one finds that for each $\lambda \in \rho(x, T)$, $x=u(\lambda)-T u(\lambda) \in M$, so that $J x=x$. Now, operating on both sides of equation (4.5) by $J$, using the fact that $J$ commutes with $T$, and hence that $J^{*}=J$ commutes with $T^{*}$, and writing $\widehat{u}=J w$, we find that $\hat{u}$ satisfies the fundamental equation

$$
x=\bar{\lambda} \hat{u}(\lambda)-T^{*} \widehat{u}(\lambda),
$$

for each $\lambda \in \rho(x, T)$. As $J \in B(X)$, $\hat{u}$ is analytic, and thus is an extended resolvent of $T^{*}$ at $x$ with $\rho(x, T) \leqq \bar{\rho}\left(x, T^{*}\right)$. Therefore $\sigma(x, T) \supseteqq \bar{\sigma}\left(x, T^{*}\right)$.

As for the second part of the theorem, suppose that $\hat{T}$ is the minimal closed extension of $T$. We show first that $\sigma(x, \hat{T})=\sigma(x, T)$. Suppose therefore that $\alpha \in \rho(x, \hat{T})$, then $R(\cdot, \hat{T}) x$ has an analytic 
continuation to $\alpha$. By the definition of analyticity, and the Riesz representation theorem, this is equivalent the statement that $(y, R(\cdot, \hat{T}) x)$ has this continuation for all $y \in X$. Now observe that for $\lambda \in \rho(\hat{T})$ the following chain of equalities hold: $(y, R(\lambda, \hat{T}) x)=$ $\left(R(\lambda, \widehat{T})^{*} y, x\right)=\left(R\left(\bar{\lambda}, T^{*}\right) y, x\right)=\left(R\left(\lambda, T^{*}\right) y, x\right)=(y, R(\lambda, T) x)$, where we have used the fact that $\hat{T}^{*}=T^{*}$. From the above this therefore shows that $\alpha \in \rho(x, T)$, and hence that $\sigma(x, \widehat{T})=\sigma(x, T)$ for all $x \in X$. Now, in general $\hat{T}=T^{* *}$, and as $M\left(x, T^{*}\right)$ reduces $T^{* *}$, the first part of the theorem applies to show that $\sigma\left(x, T^{*}\right) \supseteqq \bar{\sigma}\left(x, T^{* *}\right)$. Therefore, $\sigma(x, T) \supseteqq \bar{\sigma}\left(x, T^{*}\right) \supseteqq \sigma\left(x, T^{* *}\right)=\sigma(x, \hat{T})=\sigma(x, T)$, and the second conclusion now follows.

Corollary 4.6. If $T \in B(X)$, and if $\{u(\lambda): \lambda \in \rho(x, T)\}$ is fundamental in $X$, then $\sigma(x, T) \supseteqq \bar{\sigma}\left(x, T^{*}\right)$.

When $X$ is only a Banach space we can prove the following results, the proof coming directly from Theorem 2.5 and the remarks that introduced this section.

THEOREM 4.7. Suppose that $T \in B(X)$ and that $X_{0} \subseteq X$ and $X_{0}^{*} \subseteq X^{*}$ are subspaces of the second category, then $\bigcup_{x \in X_{0}} \sigma(x, T)=$ $\bigcup_{x^{*} \in X^{*} 0} \sigma\left(x^{*}, T^{*}\right)$.

Now let $\pi: X \rightarrow X^{* *}$ be the cannonical isomorphism of $X$ into its bi-dual space $X^{* *}$. Then, with this notation in mind, we present the following slight improvement of Theorem 4.7.

Theorem 4.8. Suppose that $T$ is densely defined in $X$ and that $\rho(T) \neq \phi$. Let $\Gamma^{*} \leqq \pi(X)$ be a determining manifold for $X^{*}$, and let $X_{0}=\pi^{-1}\left(\Gamma^{*}\right)$. Then if $\Gamma$ is a determining manifold for $X$, $\bigcup_{x \in X_{0}} \sigma(x, T)=\bigcup_{x^{*} \in \Gamma} \sigma\left(x^{*}, T^{*}\right)$.

Proof. We show first that the image $\pi(X)$ does in fact contain a determining manifold $\Gamma^{*}$ for $X^{*}$, in fact $\pi(X)$ itself is such a manifold. For, by the Hahn-Banach theorm, for all functionals $x^{*} \in X^{*}$, we have $\left\|x^{*}\right\|=\sup \left\{\left|x^{*} x\right|:\|x\|=1, x \in X\right\}$, and so, with $x^{* *}=$ $\pi(x)$, we find that $\left\|x^{*}\right\|=\sup \left\{\left|x^{* *} x^{*}\right|:\left\|x^{* *}\right\|=1, x^{* *} \in X^{* *}\right\}$, as $\pi$ is an isometry. Again because $\pi$ is isometric, $\pi(X)$ is a closed subspace of $X^{* *}$, and therefore is a determining manifold for $X^{*}$. Suppose now that $\alpha \in \bigcap_{x \in X_{0}} \rho(\mathrm{x}, T)$, so that for each $x \in X_{0}, R(\cdot, T) x$ has an analytic continuation to a neighbourhood of $\alpha$. Thus, for each $x^{*} \in \Gamma$, the complex-valued function $x^{*}(R(\cdot, T) x)$ has this continuation property. Further, for all 


$$
\begin{aligned}
\lambda \in \rho(T) & =\rho\left(T^{*}\right), x^{*}(R(\lambda, T) x)=\left(R(\lambda, T)^{*} x^{*}\right) x \\
& =\left(R\left(\lambda, T^{*}\right) x^{*}\right) x=x^{* *}\left(R\left(\lambda, T^{*}\right) x^{*}\right),
\end{aligned}
$$

and so $\alpha \in \bigcap_{x^{*} \in \Gamma} \rho\left(x^{*}, T^{*}\right)$. A similar argument yields the reverse inclusion, and from these the result follows.

As a consequence of Corollary 2.6 and Theorem 4.8, we have the

Corollary 4.9. If $X$ is the dual of a Banach space $Y$, and if $T \in B(X)$ is the adjoint of an element of $B(Y)$, then $\sigma(T)=\bigcup_{x \in \Gamma} \sigma(x, T)$ for some determining manifold $\Gamma$ for $Y$.

We shall now embark on an investigation of the variation of the local spectra of $T \in B(X)$ at $x \in X$. The set function $\psi$ from $X$ to the set of subsets of $C$ will be called upper semi-continuous at $a \in X$ if, given any neighbourhood $\Omega$ of 0 , there is an $\varepsilon=\varepsilon(\Omega)>0$ such that $\psi(z) \leqq \psi(a)+\Omega$ for all $z \in X$ for which $\|z-a\|<\varepsilon$. $\psi$ is lower semi-continuous at $a$ if instead, we have $\psi(a) \leqq \psi(z)+\Omega$ for all $\|z-a\|<\varepsilon$. $\psi$ will be continuous at $a$ if it is both upper and lower semi-continuous there.

Theorem 4.10. For each $x \in X$, the map $T \rightarrow \sigma(x, T)$ is upper semi-continuous on $B(X)$.

Proof. Let $x \in X$ be fixed, and suppose that $A$ is an arbitrary endomorphism on $X$. We shall show that, with the above notation, the mapping $T \rightarrow \sigma(x, T)$ is upper semi-continuous at $A$. Let $\Omega$ be an open set containing the local spectrum of $A$ at $x$. Then, if $\hat{C}$ is the extended complex plane, with the usual topology, $\hat{\Omega}=\widehat{C} \backslash \Omega$ is compact in $\hat{C}$. Suppose that $u$ is an extended resolvent of $A$ at $x$ in $\rho(x, A)$, then, as $u$ is analytic, it is certainly strongly continuous in $\hat{\Omega}$, and is thus bounded in norm by some constant $M>0$ in $\hat{\Omega}$. Now, it is shown in [5], page 127, that if $\lambda \in \rho(A)$, the series

$$
\sum_{0}^{\infty}(T-A)^{n} R(\lambda, A)^{n+1}
$$

converges to the resolvent of $T$ at $\lambda$, provided $T$ is sufficiently close to $A$. Let $u_{n}$ be an analytic continuation of $R(\cdot, A)^{n+1} x$ into $\sigma(A)$, then, because of (4.6), if the series

$$
\sum_{0}^{\infty}(T-A)^{n} u_{n}
$$

converges, it will converge to an analytically extended resolvent of $T$ at $x$. Because of equation (4.7.6) of [12], we see that 


$$
u_{n+1}(\lambda)=(-)^{n} u^{(n)}(\lambda) / n !
$$

for all $\lambda \in \rho(x, T)$, and $n=0,1,2, \cdots$.

We may suppose that $\sigma(x, A) \neq \sigma(A)$, as in this case, Theorem 5.2.3 of [5] applies to show that the map $T \rightarrow \sigma(T)$ is upper semicontinuous on $B(X)$. We thus restrict attention to the compact set $\hat{\Omega} \backslash \rho(A)$. Choose a covering of $\hat{\Omega} \backslash \rho(A)$ by open $\operatorname{discs}\left\{\Delta\left(a_{\kappa}, R_{k}\right)\right\}$ which lie in $\rho(x, A)$. Then there is a finite subcovering of $\hat{\Omega} \backslash \rho(A)$, say

$$
\left\{\Delta\left(a_{\kappa}, R_{\kappa}\right): \kappa=0,1, \cdots, N\right\}
$$

and in each of these discs we have the estimates $\left\|u^{(n)}(\lambda)\right\| \leqq M R_{\kappa}^{-n} n$ ! if $\lambda \in \Delta\left(a_{\kappa}, R_{\kappa}\right)$. Choose $R=\max \left(R_{0}, R_{1}, \cdots, R_{N}\right)>0$, then, because of equation (4.8), $\left\|u_{n+1}(\lambda)\right\| \leqq M R^{-n}$ for all $\lambda \in \hat{\Omega} \backslash \rho(A)$. Therefore, the series (4.7) converges to an extended resolvent of $T$ at $x$, for all $T$ which satisfy $\|T-A\|<\varepsilon=1 / R$, and for these $T, \rho(x, T) \supseteqq \hat{\Omega}$. That is, $\sigma(x, T) \subseteq \Omega$, and the proof is complete.

Examples can be given which shown that the map $T \rightarrow \rho(x, T)$ is not continuous on $B(X)$, (cf: Newburgh [6]), and so it is natural to ask where this map is in fact continuous. To give a partial answer to this question we let $B_{0}(X)$ be a closed sub-algebra of $B(X)$, and denote by $Z_{0}(X)$ the centre of the algebra $B_{0}(X)$. Then

Corollary 4.11. For each $x \in X$, the map $T \rightarrow \sigma(x, T)$ is continuous on $Z_{0}(X)$.

Proof. As $B_{0}(X)$ is closed in $B(X)$ it is obvious from Theorem 4.10 that the restriction of the above mapping to $B_{0}(X)$ is upper semicontinuous. We complete the proof by referring to Theorem 4 of [6].

Now let $J$ be the projection in Example 3.1, and choose a sequence $x_{n}=y_{n}+z_{n}$, with $y_{n} \in M$, no $y_{n}$ zero, and $y_{n} \rightarrow y \neq 0 ; z_{n} \in N$, no $z_{n}$ zero, and $z_{n} \rightarrow 0$ as $n \rightarrow \infty$. Then for each positive integer $n$, $\sigma\left(x_{n}, J\right)=\{0,1\}$, whilst $\sigma(y, J)=\{1\}$. Hence $\lim \sup _{n \rightarrow \infty} \sigma\left(x_{n}, J\right)$ is not contained in $\sigma(y, J)$. Therefore the map $x \rightarrow \sigma(x, T)$ is, in general, not upper semi-continuous on $X$.

Next choose $T=U^{*}$ to be the (left) unilateral shift of Example 3.2 , and let

$$
x_{n}=\left(1, \frac{1}{2}, \frac{1}{3}, \cdots, \frac{1}{n}, 0,0, \cdots\right)
$$

so that

$$
x_{n} \rightarrow x=\left(1, \frac{1}{2}, \frac{1}{3}, \cdots\right) \in l_{2} \text { as } n \rightarrow \infty \text {. }
$$


Then, for all $w=\left(w_{1}, w_{2}, \cdots\right) \in l_{2}$, we have, from equation (2.1), that

$$
\left(R\left(\lambda, U^{*}\right) x_{n}, w\right)=\lambda^{-1} \sum_{0}^{\infty} a_{n, m} \lambda^{-m},
$$

where $a_{n, m}=\sum_{0}^{n-m} \bar{w}_{k} /(m+k)$ if $m<n$; and $a_{n, m}=0$ if $m \geqq n$. Therefore $\sigma\left(x_{n}, U^{*}\right)=\{0\}$. But, at least initially for $\lambda \in \rho\left(U^{*}\right)$,

$$
\left(R\left(\lambda, U^{*}\right) x, w\right)=\lambda^{-1} \sum_{0}^{\infty} a_{m} \lambda^{-m},
$$

where $a_{m}=\sum_{0}^{\infty} \bar{w}_{k} /(m+k)$. $w$ being an arbitrary vector in $l_{2}$, we choose $w=x$, and then we find that

$$
a_{m}=\left(1+\frac{1}{2}+\cdots+\frac{1}{m}\right) / m \sim(\gamma+\log m) / m \text { as } \quad m \rightarrow \infty ;
$$

here $\gamma$ is Euler's constant. Hence the function represented by the series (4.9) has a singularity on the boundary of the unit disc, and so cannot be continued analytically to every point of the complex plane punctured at the origin. So $\lim \inf _{n \rightarrow \infty} \sigma\left(x_{n}, U^{*}\right)$ does not contain $\sigma\left(x, U^{*}\right)$, and thus the map $x \rightarrow \sigma(x, T)$, is not, in general, lower semi-continuous on $X$.

Now choose $T=U$ again. Thus, for each non-zero $x \in l_{2}$, the local spectrum at $x$ is $\sigma(U)$, and therefore the mapping $x \rightarrow \sigma(x, U)$ is continuous at each nonzero point of $X$. Furthermore, this gives an example of the best possible result in this direction, as, by Theorem 2.3 , this map can never be continuous at 0 , at least for $T \in B(X)$.

In spite of these negative results, we can establish the following

THEOREM 4.12. Let $x_{n} \rightarrow x$ strongly (weakly), and let $u_{n}$ be an extended resolvent of the closed (densely defined) operator $T$ at $x_{n}$. If, (i) the ranges of the $u_{n}$ lie in some compact (weakly compact) set; and (ii) $u_{n}$ is bounded in some domain $E$ for which $\mathrm{Cl}(E) \subseteq$ $\bigcap_{0}^{\infty} \rho\left(x_{n}, T\right)$, then there is a subsequence $u_{n_{i}}$ converging strongly (weakly) to the extended resolvent of $T$ at $x$, in $E$.

Proof. Let $E$ be an open connected set, closure contained in the intersection $\bigcap_{0}^{\infty} \rho\left(x_{n}, T\right)$. The distance from $E$ to the local spectrum $\sigma\left(x_{n}, T\right)$ is positive, ond so $u_{n}(\lambda)$ is bounded in $\lambda$, for each integer $n$. Hence by (ii), the sequence of extended resolvents is uniformly bounded in $E$. Because of the compactness assumption (i), the Vitali theorem for vector-valued analytic functions asserts that there is a subsequence $u_{n_{i}}$ which converges to some function $u$, which is also analytic in $E$. Now, $x_{n_{i}}=T_{i} u_{n_{i}}(\lambda) \rightarrow x$ as $i \rightarrow \infty$, as $\left\{x_{n}\right\}$ converges, this being true for all $\lambda \in E$. Therefore, as $T$ is a closed operator, $u$ belongs to the 
domain of $T$, and $\lambda u(\lambda)-T u(\lambda)=x$ for each $\lambda \in E$, so that $u$ is an extended resolvent of $T$ at $x$ in $E$, and $\rho(x, T) \supseteqq E$. If we now assume that the above statements pertaining to convergence and compactness are replaced by the corresponding statements in the weak topology of $X$, then, for all $x^{*} \in X^{*}$, as the domain of $T$ is dense in $X$, $x^{*} x_{n_{i}}=x^{*}\left(T_{\lambda} u_{n_{i}}(\lambda)\right)$ for all $\lambda \in E$. But this last expression is

$$
\left(T_{\lambda}^{*} x^{*}\right) u_{n_{i}}(\lambda) \rightarrow\left(T_{\lambda}^{*} x^{*}\right) u(\lambda) \text { as } i \rightarrow \infty,
$$

and the same conclusion is valid again.

5. The local operational calculus. Among other things, the operational calculus, $([2], 566)$, allows one to define the operator $f(T)$, for a given endomorphism $T$, for a large class of functions $f$. To wit, those functions holomorphic on some neighbourhood of the spectrum of $T$. This class of functions will be denoted by $F(T)$. In a natural way we shall extend this calculus to the class $F(x, T)$ of functions holomorphic on a neighbourhood of the local spectrum of $T$ at $x \in X$. However, the domain of $f(T)$ will be the one-dimensional subspace generated by $x$.

Throughout this section, we shall assume that $T$ has the singlevalued extension property at $x$, so that in particular, the extensions of the resolvent will have no branch points in $\rho(x, T)$.

An open set $U_{x}$ will be called $T$-admissible at $x$ if (i) $U_{x} \supseteqq \sigma(x, T)$; (ii) $U_{x}$ has only a finite number of components, and (iii) the boundary Bd $U_{x}$ of $U_{x}$ consists of a finite number of closed, disjoint, rectifiable Jordan curves. Suppose that $T \in B(X)$. Then, if $f \in F(x, T)$ is holomorphic on the open set $V \supseteqq \sigma(x, T)$, there is, because of Theorem 2.3, and a lemma due to Taylor, ([9], 190), a $T$-admissible set $U_{x} \subseteq V$. We now define the operator $f(T)$ at $x$ by the integral

$$
f(T) x=(2 \pi i)^{-1} \int_{B d U_{x}} u(\zeta, x, T) f(\zeta) d \zeta,
$$

where $\zeta \rightarrow u(\zeta, x, T)$ is the extended resolvent of $T$ at $x$ in $\rho(x, T)$. It is readily seen that (5.1) is independent of the particular $T$-admissible set chosen, and thus $f(T) x$ is a well defined element of $X$.

THEOREM 5.1. (i) Under the obvious operations, $F(x, T)$ forms an algebra over $C$;

(ii) If $\left\{f_{n}\right\} \subseteq F(x, T)$ converges uniformly on $U_{x}$ to $f$, then $f \in F(x, T)$, and $\left\{f_{n}(T) x\right\}$ converges, in the strong topology of $X$, to $f(T) x$;

(iii) If $f$ can be represented by the convergent series

$$
f(z)=\sum_{0}^{\infty} a_{n} z^{n} \text { in } U_{x} \text {, then } f(T) x=\sum_{0}^{\infty} a_{n} T^{n} x ;
$$


(iv) Suppose that $f, g \in F(x, T)$. Let $h=f \cdot g$, and let $y=g(T) x$. Then if $f \in F(y, T)$ we have $h(T) x=f(T) y$.

Proof. The proofs of parts (i), (ii) and (iii) will be omitted as they follow readily from the proofs of the corresponding statements in [2], page 568. Part (iv) may also be adapted from Theorem VII 3.10 (b) of the above reference, by using the fact that if $u(\cdot, x)$ is the extended resolvent of $T$ at $x$, then for $\lambda, \mu \in \rho(x, T)$ we have $u(\lambda, x)-u(\mu, x)=-(\lambda-\mu) u(\lambda, u(\mu, x))$-a result easily derived from the first resolvent equation.

We shall now establish the following local version of the celebrated spectral mapping theorem.

Theorem 5.2. Suppose that $T \in B(X)$ and that $f \in F(T)$. Then, for all $x \in X, \sigma(x, f(T))=f(\sigma(x, T))$.

Proof. Suppose first that $\alpha \in \sigma(x, T)$, then the function $g$ defined by $g(z)=(f(\alpha)-f(z)) /(\alpha-z)$ belongs to $F(T)$, and $(\alpha 1-T) g(T)=$ $f(\alpha) 1-f(T)$. So if $f(\alpha) \notin \sigma(x, f(T))$, there would exist a function $u$, analytic at $f(\alpha)$, such that $[f(\alpha) 1-f(T)] u(f(\alpha))=x$. But this in turn would imply that $(\alpha 1-T)[g(T) u(f(\alpha))]=x$. Now define the $X$-valued function $w$ by $w(\lambda)=g(T) u(f(\lambda))$. Then as $g(T) \in B(X)$, $w$ is analytic at $\alpha$, and $\alpha w(\alpha)-T w(\alpha)=x$, which contradicts the fact that $\alpha \in \sigma(x, T)$. Therefore $f(\sigma(x, T)) \subseteq \sigma(x, f(T))$. Now, by Dunford's spectral mapping theorem, ([2], 569), and because of Corollary 2.6, we have that

$$
\begin{aligned}
\bigcup_{x \in X} \sigma(x, f(T)) & =\sigma(f(T))=f(\sigma(T))=f\left(\bigcup_{x \in X} \sigma(x, T)\right) \\
& =\bigcup_{x \in X} f(\sigma(x, T)) .
\end{aligned}
$$

But because we have already shown that $f(\sigma(x, T)) \subseteq \sigma(x, f(T))$, this certainly implies that $\sigma(x, f(T))=f(\sigma(x, T))$ for all $x \in X$.

Suppose now that $T$ is a closed (unbounded) linear transformation from its domain $D_{T} \subseteq X$ to $X$, and that for a given $x \in X, \rho(x, T)$ is not empty. Let $F(x, T)$ be the algebra of complex-valued functions holomorphic on $\sigma(x, T)$, and analytic at $\infty$. An open set $U_{x}$ will be called (locally) $T$-admissible in this case if it satisfies the same conditions as previously, except that the bounding curves need no longer be rectifiable. Then if $f \in F(x, T)$ is holomorphic on an open set $V$, we define, for any $T$-admissible set $U_{x} \subseteq V$,

$$
f(T) x=\delta f(\infty) x+(2 \pi i)^{-1} \int_{\mathrm{Bd} U_{x}} u(\zeta, x) f(\zeta) d \zeta,
$$

where $\delta=0$ or 1 according as whether $\sigma(x, T)$ is unbounded or not. 
This local operational calculus provides a generalization of the calculus due to Taylor [9], in that it remains valid even if the resolvent set of $T$ is empty.

As a final comment in this section we note that the operational calculus, as defined by (5.1), does agree with the global operational calculus, whenever both are defined. This is expressed in the

THEOREM 5.3. Suppose that $f \in F(T)$ is holomorphic on the open set $W \supseteqq \sigma(T)$. Let $V$ be open, and let $\sigma(x, T) \subseteq V \cong W$. Then $\hat{f}=$ $\left.f\right|_{V} \in F(x, T)$, and further, $\hat{f}(T) x=f(T) x$.

The proof of this theorem, being obvious from the definitions, will be omitted.

6. Solutions of abstract functional equations. Utilizing Theorem 5.1 we shall now prove a result on the summability of formal solutions of functional equations, thus extending Theorem 1 of [3]. Let $T \in B(X)$, then, for a given $x \in X$ we seek to sum the formal series

$$
\lambda^{-1} \sum_{0}^{\infty} \lambda^{-n} T^{n} x
$$

to a $X$-solution of the equation

$$
\lambda u-T u=x .
$$

Let $\subseteq$ be a regular summability method which sums the geometric series $\sum_{0}^{\infty} z^{n}$ to $1 /(1-z)$ in some set $D \cong C$. Let $\Lambda(D)$ be the set of points $\zeta \in C$ for which there exists a compact subset $V \cong D$, and a $T$-admissible set $U_{x}$ such that $U_{x} \subseteq \zeta V=\{\zeta w \mid w \in V\}$. With this notation we can now state the

THEOREM 6.1. Under the above conditions, the series (6.1) is summable (S) to a solution of (6.2), for all $\lambda \in \Lambda(D)$.

Because the proof of Theorem 6.1 is identical in nature to the proof of Theorem 1 of [3], it will be omitted.

My sincere thanks go to my supervisor, Professor G. Szekeres, for his constant help and encouragement. I would also like to thank Professor Einar Hille for his many constructive comments on this work. Finally, my thanks are due to a referee for pointing out some errors in the original manuscript. 


\section{REFERENCES}

1. N. Dunford, Survey of the theory of spectral operators, Bull. Amer. Math. Soc. 64 (1958), 217-274.

2. N. Dunford, and J. T. Schwartz, Linear operators, Vol. I., Interscience, 1964, 2nd. edition.

3. J. D. Gray, The summability of formal solutions of functional equations, J. Aust. Math. Soc. (2) 7 (1967), 141-144.

4. - Ph.D. thesis (sumbitted to U.N.S.W., 1967).

5. E. Hille, and R.S. Phillips, Functional analysis and semi-groups, Amer. Math. Soc. Coll. 31. (1958).

6. J. D. Newburgh, The variation of spectra, Duke Math. J. 18 (1951), 165-176.

7. R. C. Sine, Spectral decomposition of a class of operators, Pacific J. Math. 14 (1964), 333-352.

8. J. G. Stampfli, Analytic extensions and spectral localization, J. Math. Mech. (3) 16 (1966), 287-296.

9. A. E. Taylor, Spectral theory of closed distributive operators, Acta Math. 84 (1951), 189-224.

Received March 21, 1967.

UNiversity OF NeW SOUth Wales

Kensington, N.S.W., Australia 


\section{PACIFIC JOURNAL OF MATHEMATICS}

\section{EDITORS}

\section{H. ROYDEN}

Stanford University

Stanford, California

\author{
R. R. Phelps \\ University of Washington \\ Seattle, Washington 98105
}

\section{J. DugundJI}

Department of Mathematics University of Southern California Los Angeles, California 90007

\section{RICHARD ARENS}

University of California

Los Angeles, California 90024

\section{ASSOCIATE EDITORS}

\section{E. F. BECKENBACH}

B. H. NEUMANN

F. WOLF

K. YOSIDA

\section{SUPPORTING INSTITUTIONS}

UNIVERSITY OF BRITISH COLUMBIA CALIFORNIA INSTITUTE OF TECHNOLOGY UNIVERSITY OF CALIFORNIA MONTANA STATE UNIVERSITY UNIVERSITY OF NEVADA NEW MEXICO STATE UNIVERSITY OREGON STATE UNIVERSITY UNIVERSITY OF OREGON OSAKA UNIVERSITY UNIVERSITY OF SOUTHERN CALIFORNIA

\author{
STANFORD UNIVERSITY \\ UNIVERSITY OF TOKYO \\ UNIVERSITY OF UTAH \\ WASHINGTON STATE UNIVERSITY \\ UNIVERSITY OF WASHINGTON \\ AMERICAN MATHEMATICAL SOCIETY \\ CHEVRON RESEARCH CORPORATION \\ TRW SYSTEMS
}

NAVAL WEAPONS CENTER

Mathematical papers intended for publication in the Pacific Journal of Mathematics should be in typed form or offset-reproduced, double spaced with large margins. Underline Greek letters in red, German in green, and script in blue. The first paragraph or two must be capable of being used separately as a synopsis of the entire paper. It should not contain references to the bibliography. Manuscripts, in duplicate if possible, may be sent to any one of the four editors. All other communications to the editors should be addressed to the managing editor, Richard Arens, University of California, Los Angeles, California 90024.

Each author of each article receives 50 reprints free of charge; additional copies may be obtained at cost in multiples of 50 .

The Pacific Journal of Mathematics is published monthly. Effective with Volume 16 the price per volume (3 numbers) is $\$ 8.00$; single issues, $\$ 3.00$. Special price for current issues to individual faculty members of supporting institutions and to individual members of the American Mathematical Society: $\$ 4.00$ per volume; single issues $\$ 1.50$. Back numbers are available.

Subscriptions, orders for back numbers, and changes of address should be sent to Pacific Journal of Mathematics, 103 Highland Boulevard, Berkeley 8, California.

Printed at Kokusai Bunken Insatsusha (International Academic Printing Co., Ltd.), 7-17, Fujimi 2-chome, Chiyoda-ku, Tokyo, Japan.

PUBLISHED BY PACIFIC JOURNAL OF MATHEMATICS, A NON-PROFIT CORPORATION

The Supporting Institutions listed above contribute to the cost of publication of this Journal, but they are not owners of publishers and have no responsibility for its content or policies. 


\section{Pacific Journal of Mathematics}

\section{Vol. 27, No. $2 \quad$ February, 1968}

Leonard E. Baum and George Roger Sell, Growth transformations for

functions on manifolds ............................ 211

Henry Gilbert Bray, A note on CLT groups ................... 229

Paul Robert Chernoff, Richard Anthony Rasala and William Charles

Waterhouse, The Stone-Weierstrass theorem for valuable fields....... 233

Douglas Napier Clark, On matrices associated with generalized

interpolation problems ................................

Richard Brian Darst and Euline Irwin Green, On a Radon-Nikodym theorem for finitely additive set functions . ...................... 255

Carl Louis DeVito, A note on Eberlein's theorem..................... 261

P. H. Doyle, III and John Gilbert Hocking, Proving that wild cells exist . . . 265

Leslie C. Glaser, Uncountably many almost polyhedral wild $(k-2)$-cells in

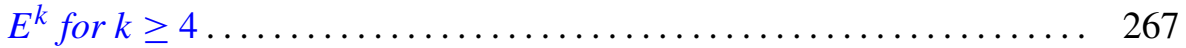

Samuel Irving Goldberg, Totally geodesic hypersurfaces of Kaehler

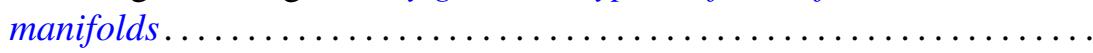

Donald Goldsmith, On the multiplicative properties of arithmetic functions .................................... 283

Jack D. Gray, Local analytic extensions of the resolvent ............ 305

Eugene Carlyle Johnsen, David Lewis Outcalt and Adil Mohamed Yaqub,

Commutativity theorems for nonassociative rings with a finite division ring homomorphic image ....................

André (Piotrowsky) De Korvin, Normal expectations in von Neumann

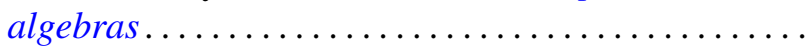

James Donald Kuelbs, A linear transformation theorem for analytic

Feynman integrals..........................

W. Kuich, Quasi-block-stochastic matrices ................... 353

Richard G. Levin, On commutative, nonpotent archimedean

semigroups ............................... 365

James R. McLaughlin, Functions represented by Rademacher series ... . . . 373

Calvin R. Putnam, Singular integrals and positive kernels............ 379

Harold G. Rutherford, II, Characterizing primes in some noncommutative

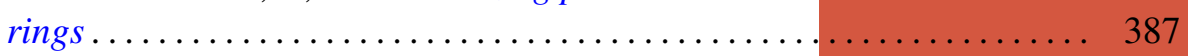

Benjamin L. Schwartz, On interchange graphs................... 393

Satish Shirali, On the Jordan structure of complex Banach *algebras . . . . . 397

Earl J. Taft, A counter-example to a fixed point conjecture............. 405

J. Roger Teller, On abelian pseudo lattice ordered groups ..... 